\title{
Diagnostic and Therapeutic Challenges of De Quervain Thyroiditis in Sub-Saharan Africa: Illustration with Two New Cases in Dakar
}

\author{
Abdoulaye Leye, Ngoné Diaba Diack, Nafy Ndiaye, Ameth Dieng, \\ Daouda Thioub, Yakham Mohamed Leye \\ Internal Medicine and Endocrinology Department, Teaching Hospital of Pikine, Dakar, Senegal \\ Email: ablayleye@hotmail.com
}

How to cite this paper: Leye, A., Diack, N.D., Ndiaye, N., Dieng, A., Thioub, D. and Leye, Y.M. (2017) Diagnostic and Therapeutic Challenges of De Quervain Thyroiditis in Sub-Saharan Africa: Illustration with Two New Cases in Dakar. Open Journal of Endocrine and Metabolic Diseases, 7 , 219-226.

https://doi.org/10.4236/ojemd.2017.712019

Received: October 10, 2017

Accepted: December 17, 2017

Published: December 20, 2017

Copyright $\odot 2017$ by authors and Scientific Research Publishing Inc. This work is licensed under the Creative Commons Attribution International License (CC BY 4.0).

http://creativecommons.org/licenses/by/4.0/

\begin{abstract}
Introduction: De Quervain's subacute thyroiditis is an inflammatory granulomatosis with clinical presentation some time atypical leading eventually to misdiagnosis or inadequate treatment. In our area, physician should be aware of this possible etiology in presence of recent and acute features of thyrotoxicosis with or without painful firm goiter. We illustrate this fact with two new observations from Sub-Saharan Africa. Observations: Our first patient is a 70-year-old Guinean woman presenting a painful nodular and hard goiter at palpation. Her history was recent in a context of deterioration of the general state with a sub clinical thyrotoxicosis syndrome. The diagnosis of probable malignant thyroid tumor was evoked initially and a thyroidectomy considered. She presented an important biological inflammatory syndrome and biological peripheral hyperthyroidism. Neck ultrasound examination showed heterogeneous patchy decreased echogenicity. The diagnosis of subacute De Quervain's thyroiditis was set up. A corticosteroid therapy at a rate of 20 $\mathrm{mg} /$ day of prednisone was begun with a fast degression of dose. After 3 weeks of treatment, we noticed total clinical recovery normalization of biological parameters. The second observation was about a 52-year-old Senegalese women living in Bamako. She presented cervical pains evolving for 2 months in a context of flu-like syndrome associated with thyrotoxicosis and marked biological inflammatory syndrome. The thyroid ultrasound showed global heterogeneity with characteristic aspect of "geography map" leading to the diagnosis of subacute thyroiditis. A combination therapy with low dose of prednisone, $\beta$-blockers and tranquillizers. We noticed fast disappearance of clinical and biological signs. At $5 \mathrm{mg}$ per day of prednisone, there were moderate signs of relapse with rapid favorable evolution after few days under increased dose of prednisone up to $10 \mathrm{mg} /$ day. Conclusion: De Quervain's thy-
\end{abstract}


roiditis can appear under misleading features opening out to make wandering diagnosis. A good clinical evaluation coupled with hormonal and ultrasound thyroid examination may help for right management. The use of low dose of corticosteroid had enabled us to obtain a fast control of main clinical and biological abnormalities.

\section{Keywords}

De Quervain's Thyroiditis, Nodular Goiter, Tropical Area

\section{Introduction}

The subacute granulomatous De Quervain's thyroiditis (DQT) is a non-purulent inflammatory lesion of thyroid gland in connection probably with a viral infection [1].

The triad cervical pain, thyrotoxicosis and inflammatory syndrome are very evocative of this affection even if the presentation can be very heterogeneous [2].

This pathology is seldom reported in our context where it set up diagnostic problems because of its atypical features but also therapeutic with badly codified strategies in the duration and posology.

We report two observations of DQT, the first in a pseudo-tumoral form and the second in presentation without goiter, to illustrate difficulties underlined above in Sub-Saharan area.

\section{Observations}

\subsection{Case 1}

A 70-year-old lady from Guinea had consulted in our department in Dakar for second medical opinion. She was followed for a nodular firm goiter associated with a deterioration of the general state. An indication for total thyroidectomy would have been set up for diagnostic and eventually therapeutic exploration. At admission in our department in August 2017, she described former cervical pains evolving for 6 weeks with painful swallowing simulating an angina in a context of flu syndrome. The patient also presented a weight loss associated asthenia and important non-selective anorexia. Moreover, she had intermittent palpitations without other classical signs of thyrotoxicosis. The blood pressure was $120 / 90 \mathrm{mmHg}$, temperature at $37.9^{\circ} \mathrm{C}$ for a heart rate up to 108 beats per minute. The physical examination found palpation feeling of firm even hard multinodular and sensitive goiter with limited move of the neck. There were also sensitive non-compressive and inflammatory sub-mandibular adenopathies under mandibulaires. The examination of the throat was normal.

Biological exploration, showed a non-specific inflammatory syndrome with high serum $\mathrm{C}$ reactive protein (CRP) up to $864 \mathrm{mg} / \mathrm{l}(\mathrm{N}<6 \mathrm{mg} / \mathrm{L})$, increased erythrocyte sedimentation rate to $65 \mathrm{~mm}$ at first hour $(\mathrm{N}<20 \mathrm{~mm})$, high serum fibrinogen level at $6.48 \mathrm{~g} / \mathrm{L}(\mathrm{NR}: 2-4 \mathrm{~g} / \mathrm{L}$ ). The complete blood count (CBC) did 
not show abnormalities. The thyroid hormonology objectified a hyperthyroidism with low serum Thyroid stimulating hormone (TSH) at $0.034 \mathrm{uUI} / \mathrm{mL}$ (NR: 0.35 - 4.94) and slightly high serum free thyroxine (fT4) to $28.05 \mathrm{pmol} / \mathrm{L}$ (NR: 9.1 - 23.8).

Ultrasound found an increased volume of thyroid gland around $28 \mathrm{~mL}$ barely vascularized, heterogeneous with patchy hypo-echogenic areas drawing an aspect of "geography map" (Figure 1).

We found also an isthmic hyper-echogenic nodular formation $(12 \times 9 \mathrm{~mm})$ with necrotic center that could be classified EU-TIRADS 2 (Figure 2).

The thyroid parenchyma was barely vascularized on continuous Doppler mode (Figure 3).

All these clinical, biological and ultrasound data, made the diagnosis of subacute De Quervain's thyroiditis set up. We started immediately daily $4 \mathrm{mg}$ dose of betamethasone for three days to stamp out the inflammatory storm. Then,

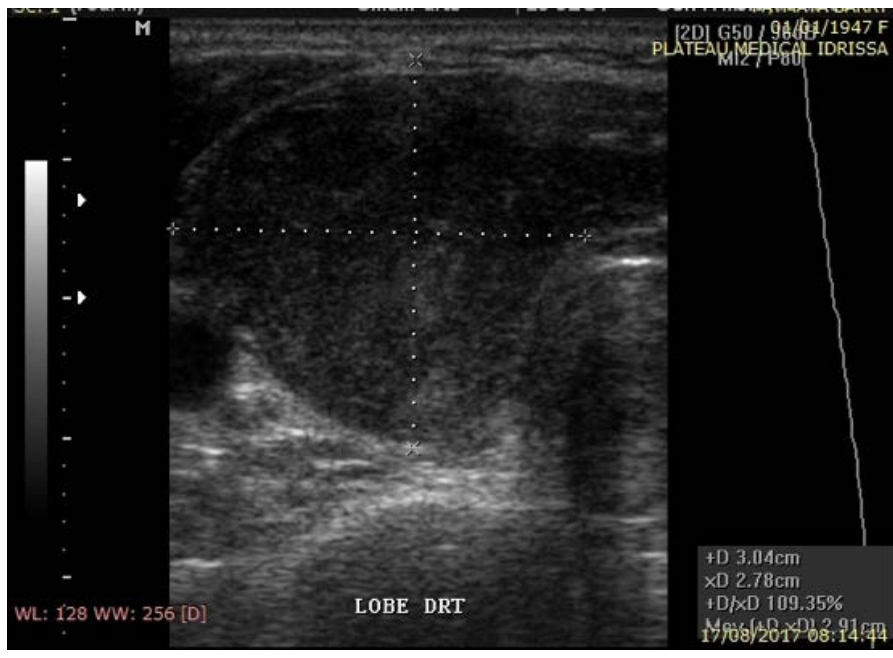

Figure 1. Heterogeneous hypo-echogenicity with geography map appearance.

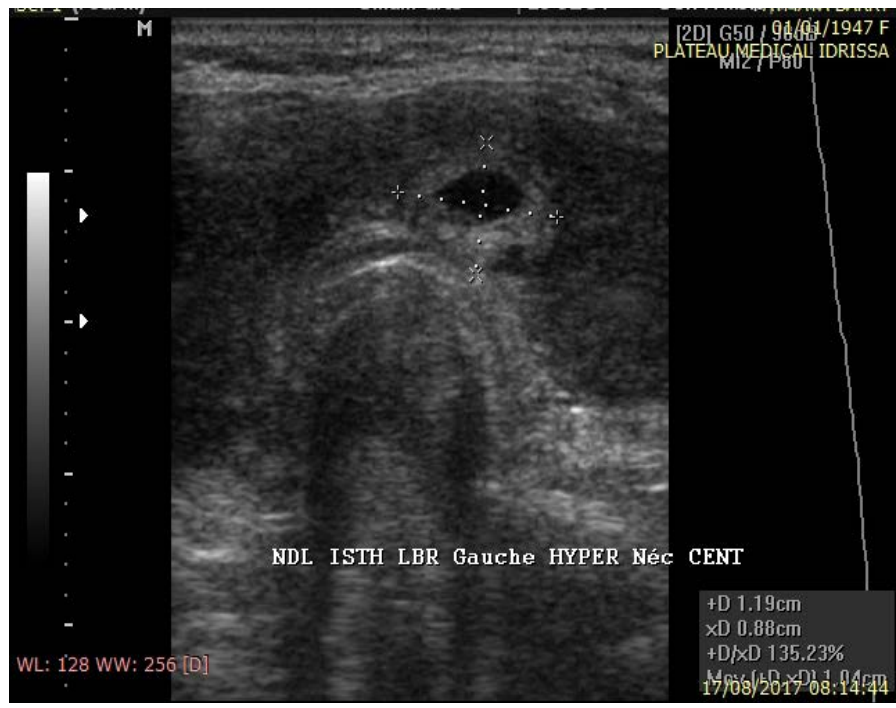

Figure 2. Isthmic hyper-echogenic nodular formation with necrotic center (EU-TIRADS 2). 

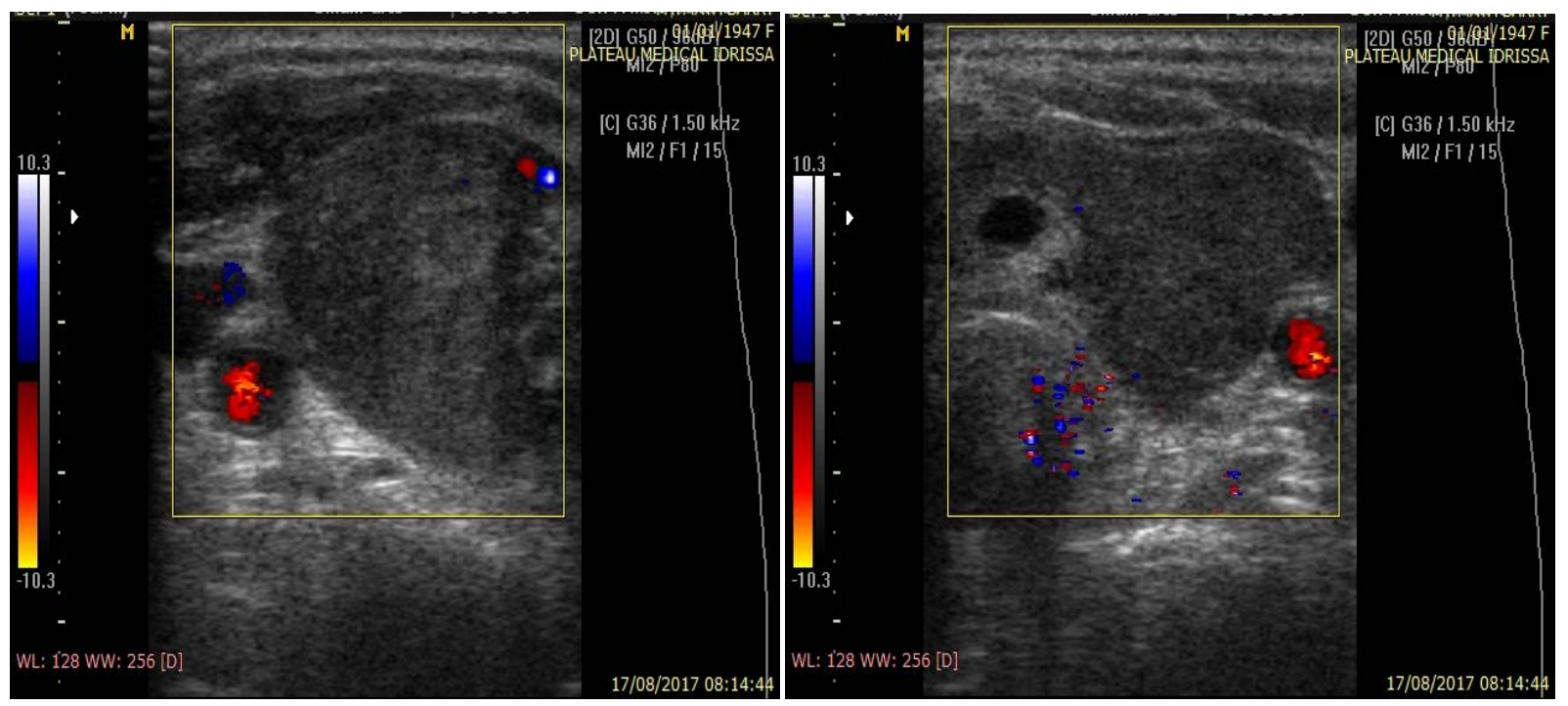

Figure 3. Sparsely vascularized thyroid parenchyma.

corticosteroid therapy relay with prednisone at the posology of $20 \mathrm{mg} /$ day was initiated with $5 \mathrm{mg}$ weekly reduction of dose, associated with propranolol over 6 weeks' duration.

The evolution was quickly favorable with amendment of the pain, of the signs of thyrotoxicosis and rapid regression of the goiter. The paraclinic examinations carried out three weeks after the beginning of the treatment found disappearance of biological inflammatory syndrome and normalization of fT4 serum level. There's no sign of relapse so far.

\subsection{Case 2}

It was about a 50 years old Senegalese housewife living at Bamako, received for anterior cervical pains evolving in the last 2 months. She complained disturbing irradiation of the pain towards her face and were not accompanied by neither throat or deglutition pain nor dyspnea. She described at the same time influenza-like syndrome made of myalgia, arthralgia, shivers and night sweating. She also brought back signs of overt thyrotoxicosis syndrome as palpitations, insomnia, irritability in a context of rapid weight loss. The physical examination highlighted a pain activated or exacerbated by palpation of the base of forehead part of neck. There was no goiter nor fever.

The biological exploration revealed a non-specific inflammatory syndrome with high rate of CRP ( $226 \mathrm{mg} / \mathrm{l}, \mathrm{N}<6 \mathrm{mg} / \mathrm{L})$, erythrocyte sedimentation rate accelerated to $44 \mathrm{~mm}$ at first hour $(\mathrm{NR}<20)$ and increase in fibrinogen with $5.40 \mathrm{~g} / \mathrm{l}$ (NR: $2-4 \mathrm{~g} / \mathrm{L})$. The rate of white cell was normal at CBC. It was also noted a peripheral hyperthyroidism with TSH ploughed up lower than 0.005 $\mathrm{uUI} / \mathrm{mL}(\mathrm{NR}: 0.35$ - 4.94) and high serum fT4 level at $39.49 \mathrm{pmol} / \mathrm{L}$ (NR: 9.1 - 23.8).

Thyroid ultrasound showed normal volume $(20 \mathrm{cc})$ of the gland whose parenchyma was heterogeneous, thinly vascularized, without nodular formation 
but hypo-echogenic areas with irregular and blurred contour, carrying out still an aspect of "geography map" of both lobes (Figure 4). There were no cervical adenopathies.

The diagnosis of subacute De Quervain's thyroiditis was thus retained for this patient. Corticosteroid therapy was then established with prednisone at initial dose of $20 \mathrm{mg} /$ day and progressive decrease over one month after three daily dose of $4 \mathrm{mg}$ of betamethasone in front of the intensity of clinical and biological inflammatory signs. Beta-blockers and tranquillizers were associated to this treatment.

The evolution was marked by a fast disappearance of the clinical signs. After 3 weeks of treatment the CRP had passed to $84 \mathrm{mg} / \mathrm{L}$, the TSH raised up to 0.05 $\mathrm{uUI} / \mathrm{mL}$ and $\mathrm{f}$ T4 had been normalized, without re-occurrence during follow up. Thyroid ultrasound control, 3 weeks after treatment found persistence of some hypo-echogenic areas looking astonishingly like somehow to a nodule that could be classified EU-TIRADS 4 (Figure 5).

\section{Discussion}

The granulomatous thyroiditis of De Quervain translates a subacute inflammatory attack often described as "influenza" of the thyroid gland [1]. Many viral factors are pointed out in the release of the inflammatory process leading to the destruction of the thyroid follicles during this process [2]. Nevertheless, for our patients we did not found proof of any ENT infection having preceded symptomatology. No viral serology had also been carried out. However, those last lab test are not being of interest in current practice [1] [2].

It is usually a pathology of the woman as for our observations with however an age of occurrence for them more advanced than in the literature (30 and 50 years) [3]

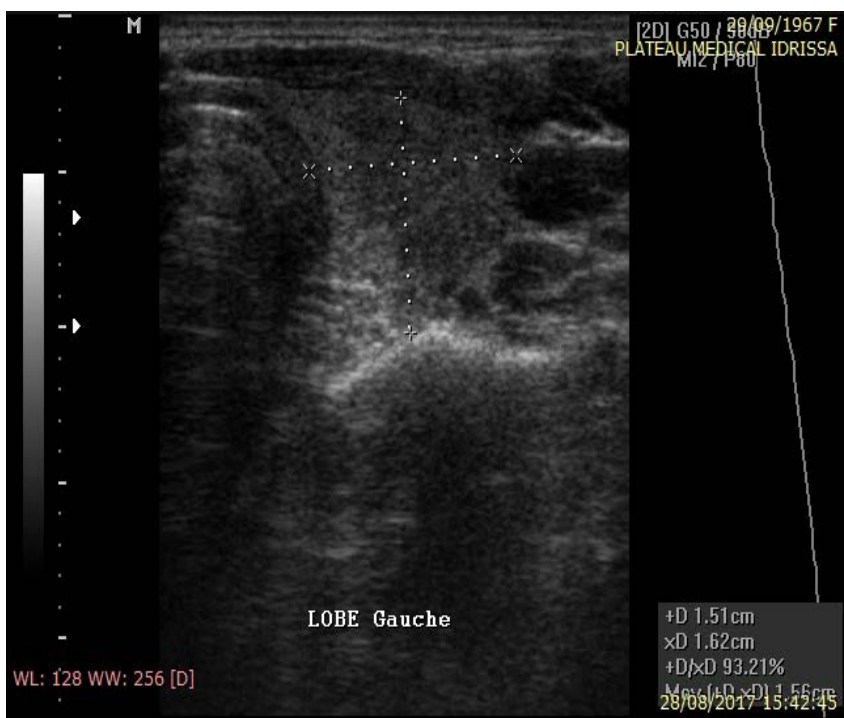

Figure 4. Thyroid ultrasound with heterogeneous parenchyma and patchy hypo-echogenic areas. 


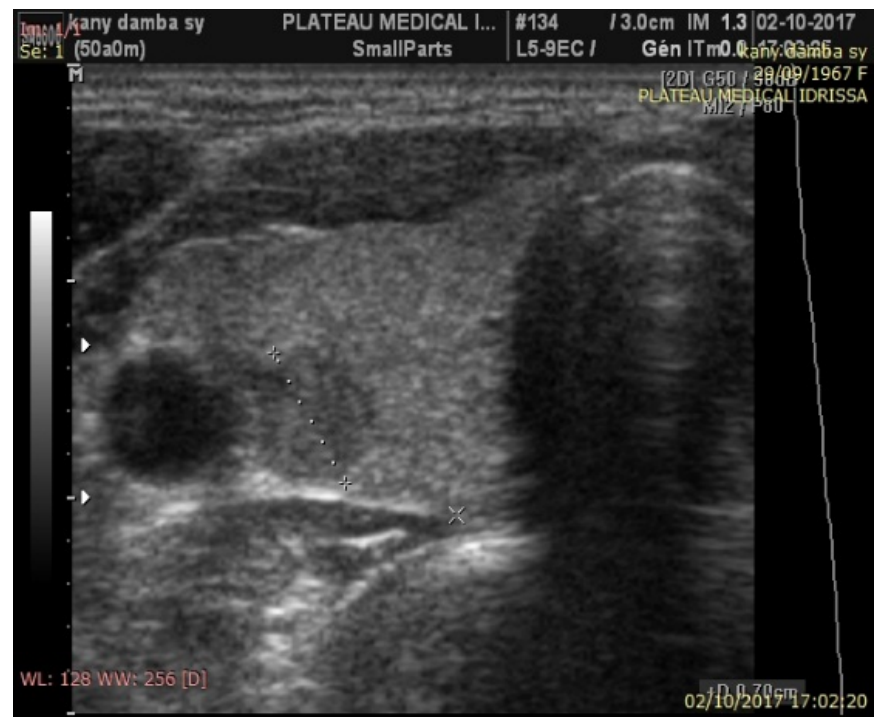

Figure 5. Thyroid ultrasound control found persistence of some hypo-echogenic areas 3 weeks after beginning of treatment.

In Africa, its real prevalence is difficult to evaluate. It would be the most frequent form of thyroiditis (51\%) according to a Cameroonian study [4]. However, each general practitioner, beyond specialist, should be aware of the possibility of this form of thyroiditis as etiology of hyperthyroidism or goiter to ovoid inappropriate excessive medical or surgical treatment.

The diagnosis of DQT is evoked in the presence of a recent cervical pain in a febrile context associated with thyrotoxicosis and biological inflammatory syndrome [1] [2]. In our second observation, this characteristic association had quickly made evoke the diagnosis. On the other hand, in the first one a wandering diagnosis was noted. Indeed, the DQT frequently induced a diagnostic concern because the initial clinical presentation is actually very polymorphic and can be misleading. Thus, in the first case, the presence of a painful recent nodular hard goiter in an elderly person associated with the weight loss had made evoke a thyroid cancer and consider the surgery. It should underline that the nodular presentation of DQT is rather rare [5] [6]. In our clinical practice, it is the second case [3]. These forms of nodular thyroiditis are misleading and expose the patients to a useless surgery for a supposed malignancy as it was the case for our first patient. In a retrospective study conducted in surgical field, 17 out of 18 cases of DQT operated after a diagnostic wandering presented a thyroid nodule [7]. However, for our patient, the very painful character of the nodule associated with the biological and especially ultrasound data had enabled us to reconsider the diagnosis of potential malignancy. Those biological data was related to the importance of the inflammatory syndrome with in particular the rise in serum fibrinogen noticed in our 2 cases. The high serum fibrinogen serum is a common demonstration of DQT found in $98 \%$ of the cases [8]. Also, it is of an interest in the differential diagnosis but also in the follow-up of the patients. The transitory hyperthyroidism is another major biological sign. It is described in the 
literature like moderate and not very symptomatic [1]. However, for our second patient, the signs of thyrotoxicosis were ahead of the picture and constituted with the cervical pains the principal reasons for consultation.

Thyroid ultrasound was of a great help to the diagnosis of DQT for us. Its positive predictive value for the diagnosis of DQT is estimated at 79.4\% [9]. The presence of hypo-echogenic areas badly delimited simulating a "map of geography" associated to a reduction of vascularization in the affected areas was the major diagnostic argument. An erasure of the thyroid limits related to oedema is reported in the nodular forms of thyroiditis but was not found in our patient [1].

The treatment of DQT is primarily medical aiming to reduce the inflammatory process. However, the therapeutic protocol is not very well codified. We had chosen in both cases for a corticosteroid therapy which showed in the literature its superiority compared to non-steroid anti-inflammatory drugs in the resolution of the symptoms [10]. Our patients had received an initial dose of $20 \mathrm{mg}$ of prednisone with a degression as after the first week of treatment. A recent prospective study showed that low dose initial of prednisone $(20 \mathrm{mg} / \mathrm{j})$ constituted an adequate and effective treatment of the DQT [11]. The exact duration of the treatment remains nevertheless to be been specified. In our patients after 3 weeks of treatment the improvement of the clinical and biological parameters was noted. However, because of the high intensity of pain and biological inflammation, as well the deep quality of life alteration, we started with three days of $4 \mathrm{mg}$ daily dose of betamethasone that induce very quick clinical improvement as similar result previously reported in another particular form [12]. It persisted nevertheless biological sub-clinical hyperthyroidism after only four weeks of treatment as well ultrasound abnormalities which are normalized usually more slowly than clinical signs [2]. A short time of treatment's maintenance (less than 3 weeks) with $5 \mathrm{mg}$ of prednisone would in addition be correlated with the risk of relapse [13].

\section{Conclusion}

The prevalence of DQT is probably underestimated in our areas. A good clinical evaluation coupled with minimal biological and thyroid ultrasound examination is necessary for the early diagnosis of this pathology even in its atypical forms. The use of corticosteroid therapy stays efficient, nevertheless, the risk of repetition with this treatment remains to be specified.

\section{References}

[1] Portmann, L. (2005) Thyroiditis: An Approach for the Practitioner. Revue Médicale Suisse, 1, Article ID: 30142.

[2] Sweeney, L.B., Stewart, C. and Gaitonde, D.Y. (2014) Thyroiditis: An Integrated Approach. American Family Physician, 90, 389-396.

[3] Leye, A., Manga, N., Ndongo, S., et al. (2004) Thyroiditis of “Quervain”: Observation of a Thyrotoxic Nodular Form. Medecine d'Afrique Noire, 51, 467-468.

[4] Nouedoui, C., Juimo, A.J., Dongmo, L., et al. (1999) Thyroiditis in the Cameroonic 
Environment: Clinical, Therapeutic and Evolving Aspects. Medecine d'Afrique Noire, 46, 199-204.

[5] Jonas, C., Bertrand, C., Michel, L., et al. (2016) Painful Thyroid Nodule, a Misleading Presentation of Subacute Thyroiditis. Acta Chirurgica Belgica, 116, 301-304. https://doi.org/10.1080/00015458.2016.1147262

[6] Li, L.X., Wu, X., Hu, B., et al. (2014) Localized Subacute Thyroiditis Presenting as a Painful Hot Nodule. BMC Endocrine Disorders, 14, 4. https://doi.org/10.1186/1472-6823-14-4

[7] Ranganath, R., Shaha, M.A., Xu, B., et al. (2016) De Quervain's Thyroiditis: A Review of Experience with Surgery. American Journal of Otolaryngology, 37, 534-537. https://doi.org/10.1016/j.amjoto.2016.08.006

[8] Ma, J., Liu, R., Wu, D., et al. (2015) Utility of Plasma Fibrinogen in the Differential Diagnosis of Thyrotoxicosis. International Journal of Clinical and Experimental Medicine, 8, 1220-1226.

[9] Frates, M.C., Marqusee, E., Benson, C.B. and Alexander, E.K. (2013) Subacute Granulomatous (de Quervain) Thyroiditis: Grayscale and Color Doppler Sonographic Characteristics. Journal of Ultrasound in Medicine, 32, 505-511.

https://doi.org/10.7863/jum.2013.32.3.505

[10] Sato, J., Uchida, T., Komiya, K., et al. (2017) Comparison of the Therapeutic Effects of Prednisolone and Non Steroidal Anti-Inflammatory Drugs in Patients with Subacute Thyroiditis. Endocrine, 55, 209-214.

https://doi.org/10.1007/s12020-016-1122-3

[11] Koirala, K.P. and Sharma, V. (2015) Treatment of Acute Painful Thyroiditis with Low Dose Prednisolone: A Study on Patients from Western Nepal. Journal of Clinical and Diagnostic Research, 9, MC01-MC03. https://doi.org/10.7860/JCDR/2015/14893.6427

[12] Li, L., Sun, L., Yu, S. and Ma, C. (2016) Increased Pertechnetate and Radioiodine Uptake in the Thyroid Gland with Subacute Thyroiditis and Concurrent Graves' Disease. Hellenic Society of Nuclear Medicine, 19, 49-52.

[13] Arao, T., Okada, Y., Torimoto, K., et al. (2015) Prednisolone Dosing Regimen for Treatment of Subacute Thyroiditis. Journal of UOEH, 37, 103-110. 